\title{
Humoral First-Line Mucosal Innate Defence in vivo
}

\author{
Carl Persson \\ Laboratory Medicine, University Hospital of Lund, Lund, Sweden
}

\section{Keywords}

Plasma exudation · Humoral innate immunity · Mucosal barrier

\begin{abstract}
Based on observations in vivo in guinea-pig and human airways, this review presents plasma exudation as non-sieved transmission of bulk plasma across an unperturbed mucosa that maintains its normal barrier functions. Several steps have led to the present understanding of plasma exudation as a non-injurious response to mucosal challenges. The implication of a swift appearance of all circulating multipotent protein systems (also including antimicrobial peptides that now are viewed as being exclusively produced by local cells) on challenged, but intact, mucosal surfaces cannot be trivial. Yet, involvement of early plasma exudation responses in innate mucosal immunology has dwelled below the radar. Admittedly, exploration of physiological plasma exudation mechanisms requires in vivo approaches beyond mouse studies. Plasma exudation also lacks the specificity that is a hallmark of biological revelations. These aspects separate plasma exudation from mainstream progress in immunology. The whole idea, presented here, thus competes with
\end{abstract}

\begin{tabular}{ll}
\hline karger@karger.com & $\begin{array}{l}\text { (o) 2020 The Author(s) } \\
\text { Published by S. Karger AG, Basel }\end{array}$ \\
www.karger.com/jin & This is an Open Access article licensed under the Creative Commons \\
Karger & $\begin{array}{l}\text { Attribution-NonCommercial-4.0 International License (CC BY-NC) } \\
\text { (http://www.karger.com/Services/OpenAccessicense), applicable to } \\
\text { the online version of the article only. Usage and distribution for com- } \\
\text { mercial purposes requires written permission. }\end{array}$
\end{tabular}

strong paradigms currently entertained in the accepted research front. The present focus on humoral innate immunity in vivo further deviates from most discussions, which concern cell-mediated innate defence. Indeed, plasma exudation has emerged as sole in vivo source of major mucosal defence proteins that now are viewed as local cell produce. In conclusion, this review highlights opportunities for complex actions and interactions provided by non-sieved plasma proteins/peptides on the surface of intact mucosal barriers in vivo.

(C) 2020 The Author(s)

Published by S. Karger AG, Basel

\section{Introduction}

The airways mucosa is exposed to potentially pathogenic, airborne factors. To prevent harm there are high demands on first-line mucosal defence. Traditionally, there has been emphasis on physical-physiological mechanisms highlighting roles of epithelial, mucus barriers, mucociliary clearance and coughing. In recent decades, biological capacities of local airway cells have received the attention. The new biology, emerging several decades ago, has made accessible an attractive focus on reductive 
details. Increasingly versatile cell biology study approaches in vitro and in vivo, the latter foremost in mice, have thus generated a wealth of intriguing molecular data. The airway epithelium in particular is claimed to produce important biologically active molecules. Cell aspects have also occupied most discussions of innate immunity forwarding explanatory schemes of molecular pathways presumed to be active in airway-lung defence and repair [1, 2]. However, whether such acknowledged mechanisms are operational in vivo in humans may remain conjectural in several instances.

Independent but important in vivo findings are not always appreciated. Several astute observations in patients in 18 and 1900s' medical history may thus have been overlooked or misunderstood although they have concerned causes and treatments of disease that now take centre stage in research. Thus, for nearly a century, Solomon Solis-Cohen's insightful report on corticosteroid efficacy in severe asthma (produced by oral intake of "adrenal substance") was ignored or misinterpreted to reflect the effect of adrenalin - similarly, the astute observations by Hyde Salter on common cold-induced asthma exacerbations were incorrectly mixed up with his, indeed, equally clear accounts of the influence of cold air $[3,4]$. As a compelling sign of current acceptance of the value of these early observations, authors are now iterating these important medical history observations citing exclusively the original reports by Solis-Cohen and Salter.

Failures to note groundbreaking in vivo data have not ceased entirely. Thus, recent dominance of reductive approaches has not remedied the situation. Indeed, already in its early days, the rapidly achieved hegemonic status of cell and molecular biology research was boosted by contentions that research on "gross" physiological processes was not needed any more [5]. Priority and demands on skills [6] in medical-biological research largely turned away from exploratory in vivo pathophysiology. In accord, concepts built on continued in vivo discoveries have struggled, been ignored or misinterpreted, especially so when deviating from leading concepts built on cell and molecular biology data. Several such examples concerning activation and fates of eosinophils, epithelial barrier aspects and roles in disease of epithelium-derived molecules have recently been reviewed $[7,8]$. The present topic, plasma exudation, belongs to this list.

In vivo studies of features of plasma exudation did not fit into mainstream immunology research. In the era of reductive approaches, plasma exudation experiments required physiologically well-controlled methodologies. Further, the focus was on complex events in human or human-like, not mouse-like $[9,10]$, blood-perfused airways. The influence of an in vivo process such as plasma exudation also lacked the commonly desired specificity of immune-mechanisms. It rather represented the opening of a Pandora's box. Thus, development of data and concepts in this in vivo field has dwelled below the radar and has rarely been discussed in the immunology literature [7].

Here I will review in vivo observations in guinea-pig and human airways pertinent to early humoral innate defence. The process of plasma exudation takes centre stage. Hence, the airway epithelium and the subepithelial microcirculation are involved as co-operative mucosal end organs. Data and associated concept developments during 4 decades are presented in order to arrive at the definition of first-line defence opportunities of airways exudation of bulk plasma. Improved understanding of epithelial transmission of plasma contents has been crucial for developing the present concept.

There is some interest in interactions between the major protein systems contained in circulating plasma. The focus on such intravascular reactions ranges from potential importance in severe systemic diseases $[11,12]$ to possibility for virus vector treatments to evade the complex plasma protein milieu [13]. However, the aim of this review is to provide in vivo data, including well-controlled observations in human airways, requiring that further questions be asked:

- How would it be if molecules belonging to coagulation proteins/peptides, complement systems, pentraxins, natural antibodies, fibronectins and so on, were promptly allowed to act and interact on the surface of an unperturbed mucosa that has maintained its normal barrier function?

- Similarly, how would it be if it was plasma exudation, and not local cell produce, that brought major antimicrobial peptides, such as cathelicidins, to the surface of an intact epithelial lining?

- What then about immune evasion by pathogens?

- And, if patchy epithelial loss has occurred (as in asthma) - how would it be if exuded plasma, rather than local cell-derived molecules now in vogue, would account for the biologically active milieu that promotes epithelial regeneration in vivo?

- As with other potent defence/repair mechanisms, plasma exudation cuts both ways - how then may exaggerated plasma exudation responses contribute to disease development? 


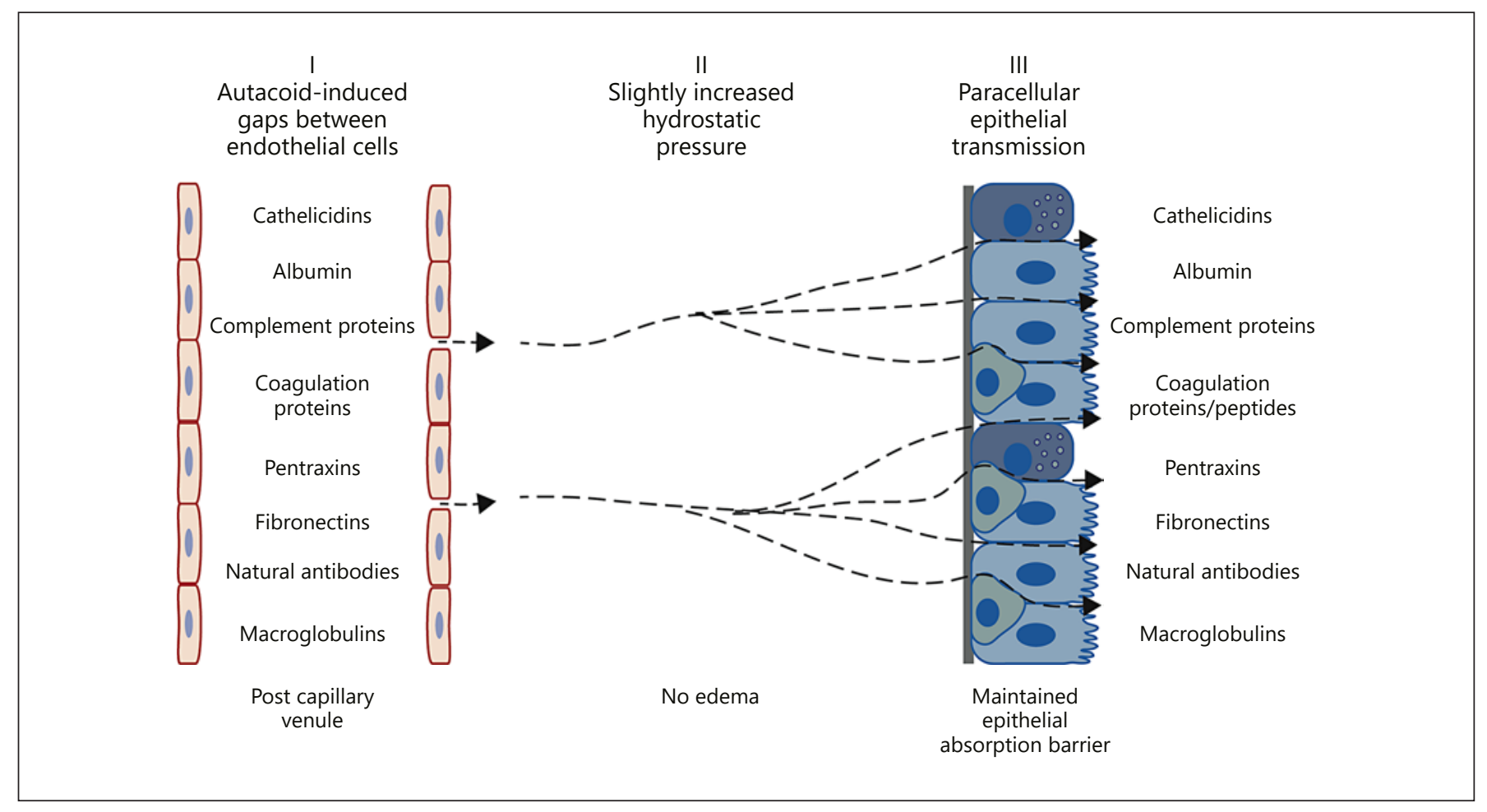

Fig. 1. Topical airway challenges with non-injurious autacoids cause exudation of bulk plasma, which is characterized by a maintained ratio between small (albumin) and large (alpha2-macroglobulin) plasma proteins in airway surface liquids as in the circulation. By this swift, humoral mechanism the entire range of circulating plasma molecules are moved to the surface of an intact mucosa. Mucosal oedema is not induced and, despite letting

\section{A Perfect Location for Providing the Airway Mucosa with Molecules and Cells}

Observations discussed in this review concern airways with a mucosa that receives a profuse, superficial blood supply. All along the human nasal and tracheobronchial tree down to the respiratory bronchioles and just beneath the epithelial lining runs an extensive micro-circulation carrying systemic oxygenated blood. It is perfectly distributed and juxtaposed to provide the mucosa of large and small airways with defence leukocytes at any location needed. What seems little realised is the equally perfect position of this microvasculature to provide the airway mucosa, and its intact (sic!) surface, with the potent innate immunity components of the entire plasma protein systems (Fig. 1).

The subepithelial bronchial microcirculation should not be confused with the pulmonary microcirculation that does not extravasate plasma in response to hista- through non-sieved plasma macromolecules, the barrier function of the airway mucosa against inhaled molecules is not reduced. Reflecting the asymmetry and plasticity of epithelial barrier cells, a slight increase in basolateral hydrostatic pressure produces such unidirectional paraepithelial perviousness. Plasma-derived molecules thus have ample opportunities to participate in first line innate defence of the intact mucosa in vivo. 
hyperresponsiveness in the allergen-challenged mice [18]. However, this does not mean that the mouse model represents asthma as well. A bleeding response further disqualifies this shape of plasma exudation in mice as a component of the first-line defence opportunities discussed in this review. The risk of bleeding from the pulmonary circulation is also a caveat in interpretation of effects of agents such as activated protein $C$ in mouse models of allergic lung inflammation [19]. Acute plasma exudation responses have not been extensively studied in mouse large airways. Allergen challenge and mediators including serotonin produced a small degree of extravasation in mouse trachea, but epithelial transmission of plasma could not be detected [20].

Perhaps reflecting the limitation of mouse models, current discussions of early local innate immune defences in the lung in vivo do not even mention the possibility of contributions of microcirculation-derived molecules (e.g., $[1,2])$.

Here I will focus on in vivo observations in guinea-pig and human airways. I will discuss challenges and mechanisms that bring all circulating plasma proteins to the surface of an otherwise tight epithelial lining in vivo. Although it is rarely considered as such in current literature, this humoral process emerges as a major first-line mucosal innate defence response.

\section{Key Events Leading to a Novel Concept of Plasma Exudation}

Experimental in vivo work during the final decades of the 20th century and onwards produced data that challenged and continue to challenge, prevailing notions of airway epithelial barrier function in health and disease. Major steps in this development, especially as regards to the fate of extravasated plasma proteins, are summarised below.

\section{New Idea of Drug Action in Asthma Linked with}

\section{Traditional Views on Plasma Exudation}

An initiating event was the discovery of beta receptormediated attenuation of extravasation of plasma evoked by presumed mediators asthma $[15,21]$. This action of the most common anti-asthma drug class contributed to a resurgence of interest in pathogenic roles of plasma exudation in this disease [22]. On the one hand, the specific microvascular anti-permeability action of beta agonists strengthened the understanding of extravasation of plasma macromolecules as a physiologically well-regulat- ed endothelial cell separation in post-capillary venules [15]. On the other hand, inhibition of plasma exudation received prompt attention as a desired drug action in asthma [22]. Other anti-asthma drugs than beta agonists, including chromones and glucocorticoids, fittingly reduced plasma exudation responses as well [22].

Although once thought of importance, it now it appears that the vascular antipemeability action of antiasthma drugs is quite limited in active disease [23-25]. Alternatively, as in the case of glucocorticoids, reduced plasma exudation in human diseased airways reflects reduced overall inflammation. Thus, topical glucocorticoid treatment has not prevented acute histamine-induced plasma exudation response [26]. These aspects are of interest with regard to basic roles of plasma exudation as a first-line defence in patients treated with the anti-asthma drugs. Treated patients would still be able to mount airways plasma exudation responses in early mucosal defence.

The movement of plasma into airway surface liquids in asthma has long been viewed as a sign and cause of oedema, epithelial shedding, airway plugs and bronchial hyperresponsiveness. These features are also considered hallmarks of asthma. However, uncertainties and inconsistencies required further studies. A major aspect concerned poor understanding of epithelial transmission of extravasated plasma in health and disease.

\section{A Puzzling Lack of Oedema}

Challenging the mucosal surface with vasoactive autacoids, expectedly, produced marked extravasation of plasma proteins from the subepithelial microcirculation in guinea-pig tracheobronchial airways. However, mucosal oedema did not result.

Traditionally, locally produced mediators such as histamine, platelet activating factor, bradykinin, serotonin and leukotrienes are classified as autacoids to indicate that they are involved in self-cure (= translation of the word autacoid). These autacoids are vascular permeability agents well known to target postcapillary, venular endothelium causing reversible separation of endothelial cells $[16,27]$. Through the ensuing endothelial gap formations non-sieved macromolecules escaped the circulation. Yet, even with marked plasma extravasation, actual oedema failed to appear [28-30]. Plasma proteins stained the challenged mucosal tissues, but this was not oedema [7]. No visual or measurable thickening of the lamina propria occurred, and there was no detectible increase in protein lymphatic transport following acute challengesinduced plasma extravasation [30]. 
In airways exposed to vascular permeability agents, failures to produce airway mucosal oedema became a tantalizing phenomenon. Bronchial oedema was, and is, such an accepted fundamental feature of asthma. Lack of oedema thus appeared to be a major limitation of the employed in vivo methodology. However, arguing against true oedema in asthma this feature has not been well supported by histology. Furthermore, an apparent swelling of the mucosa, as viewed through the bronchoscope in asthma, could just as well reflect constriction of the surrounding smooth muscle layers [7]. Indeed, instead of reflecting a flawed methodology, lack of experimentally induced oedema emerged as an important observation.

Transmission of Extravasated Plasma across an Intact Epithelial Lining Was Not Heard of

The possibility of transepithelial escape of extravasated plasma in airways with an intact mucosa was not a given consideration. First, there was no current concept or research paradigm that suggested the possibility of such an event in healthy airways. Second, the vascular permeability-active agents were not known to affect epithelial permeability. Third and in concord, in challenged airways exhibiting plasma extravasation the integrity of the epithelial lining had remained unperturbed [31-33]. Neither during nor following plasma extravasation responses were there signs of epithelial derangement. Still, despite everything that argued against it, the possibility of elimination of extravasated bulk plasma across a normally tight airway mucosa had to be addressed.

Non-surgical methods, where the airway mucosa was untouched and had maintained its normal physiological baseline, had been developed for autacoid challenges of a defined tracheobronchial mucosal surface in vivo [34]. Such a method was complemented with a selective lavage of the surface area of interest [35]. This relatively simple, additional experimental procedure provided the answer. In short, it turned out impossible to find topical mucosal challenges (amongst autacoids, allergens, occupational asthma chemicals) that produced extravasation of plasma without the plasma being moved further to the mucosal surface. Independent of size and charge, the plasma protein tracers appeared on the intact mucosal surface as a graded challenge-dependent response [35]. The plasma macromolecules moved between and all around the pseudostratified epithelial cells in the challenge area [32]. Plasma exudation measurements also revealed that an exudative hyper-responsiveness developed in airways exposed to very low levels of a major inducer of asthma such as toluene-diisocyanate [36]. In response to non-injurious challenges, the time courses of plasma exudation generally mimicked the extravasation of plasma albeit with a slight delay [37]. The epithelial passage could be speeded up somewhat by challenging with agents such as $\mathrm{H}_{2} \mathrm{O}_{2}$ that clearly injured the epithelial lining [38].

Problem apparently solved. Oedema was not allowed to develop because of an efficient paraepithelial movement of extravasated plasma to the surface of the mucosa $[35,39]$. But how could this epithelial phenomenon be explained?

\section{A Slight Increase in (Baso-)Lateral Epithelial}

Hydrostatic Pressure Moves Macromolecules across the Epithelial Lining

Plasma extravasated from subepithelial microvessels will, as it seems, automatically be transmitted to the epithelial surface. Such in vivo observations suggested the possibility that the extravasated plasma itself was causative. Could hydrostatic pressure changes, presumably caused by extravasated liquids, be involved? The slight delay between extravasation and epithelial transmission was considered consistent with this idea. The hypothesis was tested using intact tubes of airways where serosalsubepithelial and intraluminal-mucosal hydrostatic pressure was separately regulated. The guinea-pig trachea was suited for these experiments. Beside similarities to human bronchi with regard to mucosal anatomy and responsiveness, it was well reinforced by cartilage. Thus, hydrostatic pressure-induced confounding tissue distortions were avoided. The latter aspect excluded the employment of epithelial cell cultures. Accordingly, the same group that eventually confirmed our findings of a sensitive hydrostatic pressure-regulated, non-injurious basal-to apical tracheal movement of plasma macromolecules [33] had previously completely failed to produce such an effect in cell culture experiments [40]. Most likely this aspect needs consideration in future studies of junction protein mechanisms involved in the present paracellular epithelial transmission of macromolecules. Cell culture data demonstrating that epithelial junction proteins may exhibit reversible changes in response to basal to apical hydrostatic pressure increases [41] are encouraging. However, specific studies are needed involving test systems where plasma exudation-like epithelial passage of macromolecules is mimicked.

Using the tube set-up, it was discovered that a minimal rise of a few $\mathrm{cm} \mathrm{H}_{2} \mathrm{O}$ in subepithelial hydrostatic pressure was sufficient to move macromolecules to the mucosal surface. Similar to actual features of plasma exudation, the pressure-induced macromolecular transmission was 
reversible and well repeatable $[42,43]$. This observation also indicated that neither the small increase in hydrostatic pressure nor the ensuing macromolecular transmission caused significant derangement of the integrity of the epithelial lining. In accord, epithelial histology remained intact $[42,43]$.

Increasing the hydrostatic pressure on the lumen aspect of the epithelial lining did not produce macromolecular epithelial transmission. The intact airway tube experiments thus revealed the importance of producing a hydrostatic impact on the lateral aspect of columnar epithelial cells, which would be reachable only from beneath $[42,43]$. The hypothesis of an automatic coupling between extravasation and epithelial transmission was further consistent with lack of effects on the epithelial passage of macromolecules by agents known to cause plasma exudation [43]. All in all, the tube experiments mimicked salient features of plasma exudation in vivo and suggested what mechanism was involved.

\section{The Intact Epithelial Lining Exhibits Valve-Like Properties}

A big task concerned whether the plasma exudation process would also allow molecules dwelling on the mucosal surface to enter mucosal tissues? According to the established view, occurrence of airways plasma exudation is thought to be a perfectly valid index of a generally pervious epithelial lining $[7,44]$. However, if true, such bidirectional permeability would erode much of the attraction of plasma exudation as a first-line mucosal defence.

A major observation in guinea-pig airways, therefore, was that the acute autacoid-induced plasma exudation response occurred without altering the absorption permeability of the mucosa in the area of interest $[30,45]$. Underscoring its physiological nature, neither a defect mucosalabsorption barrier nor changes in epithelial structure were detected in association with plasma exudation in vivo.

In crucial similarity to the in vivo observations, the singularly outward direction of epithelial perviousness to macromolecules was equally evident in the set-up of intact airway tubes [43]. Hence, the hydrostatic pressuredriven, basal to apical, transepithelial crossing of macromolecules did not change the tight appearance of the epithelial lining cells nor did it increase the epithelial permeability to tracer molecules dwelling on the mucosal surface [43].

The in vivo observations and the observations made in intact airway tubes jointly defined an epithelial mechanism of plasma exudation. Functionally, unidirection- al, paracellular epithelial transmission of macromolecules unto the airway surface reflected a valve-like function of cell junctions of pseudostratified airway epithelium. In accord, a sensitive hydrostatic pressureregulated, hydraulic epithelial mechanism operated in a singularly outward transmission of macromolecules. This mechanism was likely activated in vivo by the extravasated plasma itself without the need of additional stimuli (Fig. 1). Taken together, the in vivo observations and the features of a plausible mechanism indicate that the outward epithelial passage of plasma macromolecules occurs without allowing any increase in the absorption of molecules dwelling on the epithelial surface. Such data are basic pillars underpinning the view that plasma exudation qualifies for a role in first-line defence of the airway mucosa.

\section{Plasma Exudation without Affecting Mucosal} Absorption Permeability in the Gut

Unidirectional perviousness emerges as a hallmark of the early plasma exudation response demonstrating its potential as a first-line mucosal defence. Would this role apply to mucosal lined organs beyond the airways?

Being a major site, the gut mucosa was targeted in exploratory studies of challenge-induced plasma exudation. Gut and airway mucosae are distinct in several respects based on differing demands on barrier functions. The gut mucosa must withstand a milieu of more constant and dramatic challenges than the airways. It was, therefore, no great surprise to find that challenge with simple autacoids (histamine, leukotrienes, bradykinin, serotonin), which are active in the airways of guinea-pigs, rats and humans, did not cause plasma exudation when applied on the ileum mucosa of anaesthetized rats [46]. More severe modes of challenges were required.

In the ileum of sensitised rats, topical mucosal allergen challenge produced acute exudation of plasma proteins lasting up to $2 \mathrm{~h}$. Importantly, similar to airway challenges, the absorption permeability of the challenged gut mucosa did not change in association with the plasma exudation response [47]. Furthermore, as in the airways, the intestinal plasma exudation response was not associated with any sign of epithelial derangement at the ultrastructural level [46]. In further similarity to the airways, detection of increased plasma proteins in the gut lumen cannot alone be considered as compelling evidence for a generally increased mucosal perviousness.

In the current literature, there seems to be little distinction made between exudation- and absorption permeability of the intestinal mucosa. This is unfortunate 
because there are clearly conditions where plasma with its multipotent components can be moved to the intestinal surface without reducing structural integrity and without reducing the normal barrier function of the epithelial lining. Based on the demonstration of such a condition, it was proposed that plasma exudation should be considered a first-line defence mechanism of gut mucosa [46].

\section{Human in vivo Data Establish First-Line Mucosal Defence Opportunities of Exuded Plasma}

Physiologically well-controlled human in vivo studies confirm and expand the preclinical observations on airways plasma exudation (see below).

\section{Human Airway in vivo Studies of the Physiology of}

\section{Humoral Innate Immunity}

Support for a swift, mucosal challenge-induced appearance of non-sieved plasma on the intact airway surface was soon obtained from studies of human airways in vivo. In fact, the preclinical animal in vivo research discussed above and human in vivo studies frequently went on in parallel, speedily confirming and expanding the original observations made in vivo in guinea-pig tracheobronchial airways. Some features of plasma exudation were actually more readily addressed in human than in animal airways. This advantage reflected in part the relevance and utility of the nose to study airway mucosal in vivo responses in humans [48].

The work involved successful development of clinical experimental methods [49]. For example, the unilateral nasal cavity could be filled with fluid for defined periods of time. Thus, known mucosal surface areas could be exposed to controlled concentrations of challenge agents as well as tracer molecules. Further, any confounding influence of mucociliary transport was neutralized. Simultaneously, the ipsilateral nasal mucosa was effectively lavaged and, at the end of the experiment, the lavage fluid was almost quantitatively retrieved [49]. Such approaches served well-controlled studies of plasma exudation responses whilst, at the same time, absorption permeability could be assessed specifically across the mucosal surface area of interest. The nasal studies were complemented with bronchial challenge approaches with retrieval of surface materials. These involved segmental challenges followed by bronchial lavage and inhalational challenges followed by induced sputum [24, 50-52].

Epithelial Passage of Bulk Plasma
Early Plasma Exudation Responses: Short-Lasting, Reversible and Reproducible

Histamine challenges of human nasal as well as bronchial mucosa promptly brought bulk plasma proteins to the exposed surface. The plasma exudation response was dose-dependent, short-lasting and fully reversible. As best demonstrated in the human experiments it was also reproducible with brief intervals $[25,53]$. These data agreed with the guinea pig in vivo data on early plasma exudation across an unharmed epithelium.

The results of histamine challenges of human nasal and bronchial airways [24, 25] were further consistent with a brief self-abortive extravasation response demonstrated in detail in animal experiments [27]. Thus, even in continued presence of an active concentration of histamine, the acute extravasation (hence also exudation) response is aborted $[14,15,54]$. It takes additional stimuli to maintain and increase duration of acute plasma exudation responses. This would be an important facet of a powerful early defence mechanism.

Speculatively, a brief spurt of bulk plasma appearing locally on the surface should be sufficient to ward off a threatening insult. If the threat is growing in strength, additional, short-lasting exudation responses are induced.

However, if actual epithelial damage and shedding are produced, plasma exudation is much sustained [55]. Indeed, as discussed further below, local plasma exudation goes on to produce a provisional cover gel continuously defending naked epithelial basement membranes. The protective plasma exudation proceeds until a new primitive layer of flat epithelium covers the patch of denuded basement membrane. Then the plasma-derived cover gel is discarded into the lumen.

With minimal sieving, plasma solutes, at least up to the size of macroglobulins, move to the mucosal surface.

In healthy airways challenged by a simple non-injurious autacoid such as histamine, quite different-sized proteins such as albumin, fibrinogen and alpha2-macroglobulin appeared in surface liquids at almost the same concentration ratio that they had in the circulation $[25,56]$. Thus, the non-sieved nature of combined extravasation and epithelial transmission of plasma macromolecules was demonstrated in humans in vivo. The size of alpha2macroglobulin outweighs (outsizes) most plasma proteins indicating that all the important molecules of the complement-, coagulation-, pentraxin-, natural antibody- and other circulating protein systems will be jointly exuded (Fig. 1).

Also a sticky and convoluted macromolecule such as fibrinogen will clearly be part of plasma exudation re-

J Innate Immun 2020;12:373-386 
sponses in human nasal and bronchial airways $[25,50]$. Indeed, in a study involving a small number of patients with allergic asthma, the acute plasma exudation response to endobronchial allergen challenge was reflected better by fibrinogen than albumin. Due to a variably increased baseline of the less-sized albumin molecule (that may slowly diffuse through the unchallenged mucosa), only fibrinogen was significantly elevated in the bronchial washing fluids. However, in accord with the bulk nature of exudation, the 2 proteins were highly correlated after allergen challenge [50].

\section{Epithelial Plasticity and Plasma Exudation with \\ Maintained Mucosal Barrier}

Consistent with an intact epithelial lining in healthy human airways, challenge with exudative histamine concentrations did not increase epithelial permeability in the opposite, inward direction [57]. This observation confirms prior findings in guinea-pig airways in vivo and in intact airway tube preparations (see above). Taken together, in vivo data obtained in human airways strongly underpin the concept of mucosal plasma exudation as first-line innate defence.

Leukocytes, too, can squeeze between epithelial lining cells and appear on the airway surface without distorting the mucosal barrier. As reviewed elsewhere [58], this passage is vital not only for the participation of defence leukocytes on the mucosal surface - the epithelial transmission of disease-driving tissue leukocytes is a major option for a silent elimination of these cells. This pathway may even be more important than apoptosis pathways. For example, whilst evidence for occurrence of apoptosis-phagocytosis of eosinophils in vivo is lacking, preclinical and patient data compellingly demonstrate a role for transepithelial cell migration at resolution of airway eosinophilic inflammation [8]. Almost as with the perviousness to macromolecules, base to apical transepithelial direction of cell traffic in vitro occurs more readily than traffic in the opposite, apical to base, direction [59].

Although entirely different mechanisms are involved, both cell and plasma protein passages attest to a remarkable yielding ability of epithelial cell junctions when approached from beneath [6]. A combined asymmetry and plasticity of airways pseudostratified epithelial lining cells are vital for a well functioning early exudative defence to challenges arrived by inhaled air.

\section{Local Mucosal Cells May Have a Capacity to Produce Defence/Repair Molecules in vitro, but Plasma Exudation Emerges a Major Source in vivo}

Reflecting current overwhelming interests in capacity of cells in vitro to produce central proteins, local airway production of defence and repair proteins have been given roles in cases when most likely the proteins have been brought to the mucosal surface by exuded plasma.

\section{Complement}

Potential role of complement proteins in defence and airways diseases have received attention and so has the possibility that local cells are contributing to these molecules. Human in vivo studies have demonstrated that airway surface complement is increased in allergic individuals at local challenge with allergen. Without mentioning that such conditions are archetypal plasma exudation conditions such data have been used to support local cell production of complement [60, 61]. Predictably $[25,56]$ and as supported by Andersson et al. [62], plasma exudation can account for increased occurrence of complement on a challenged human mucosal surface.

\section{Fibronectins}

Capacity of airway epithelial cells to produce a major repair protein such as fibronectin has attracted considerable interest. As discussed previously [7], authors have favoured the idea that in vivo data on fibronectin reflects local production, but evidence has been lacking. Reduced epithelial production of fibronectin by diseased epithelial cells in vitro has further been used to support a widely acknowledged concept stating that there is defective epithelial repair in asthma [63]. However, I think there are obvious problems with this concept:

1 Asthma-like non-sanguineous shedding of epithelium in vivo (leaving a small patch of denuded but intact basement membrane) is promptly followed by sustained, local plasma exudation creating a milieu for speedy epithelial regeneration. Held in place by a fibrin net such a provisional barrier is continuously fed by the potent repair molecules contained in plasma, notably including fibronectin [55]. In such experimental in vivo studies, there is no sign of epitheliumsecreted fibronectin - the entire pool of fibronectin is contributed by exuded plasma [55].

2 Absorption of inhaled molecules is not increased in vivo in asthma. Yet, asthma is characterised by patchy loss of epithelium [7]. This argues for a speedy restitution of mucosal barrier function rather than the oppo- 
site. The consequence of the current paradigms of increased permeability combined with defect repair could easily end in catastrophic airway conditions of ever-increasing penetration of pathogens [7]. Hence, based on independent in vivo observations, it is hypothesised that inflammation and remodelling caused by exaggerated epithelial regeneration, not defect repair, is a factor in pathogenesis of asthma [7].

\section{Cathelicidins}

To elucidate vitamin $\mathrm{D}$ activation and antimicrobial responses in human airways, Liu et al. [64] carried out endobronchial, segmental allergen challenges in allergic individuals. In bronchoalveolar lavage fluids, obtained the following day, they observed significant increases in vitamin $\mathrm{D}$ metabolites as well as in the multipotent antimicrobial defence molecule, cathelicidin (LL-37). Importantly, they also determined plasma proteins compellingly demonstrating that the entire increases, including cathelicidin, actually reflected plasma exudation [64]. Previously, it had been established that plasma contains significant levels $(>1 \mu \mathrm{g} / \mathrm{mL})$ of cathelicidin [65]. Nevertheless, epithelial or neutrophil-produced cathelicidin are the exclusive sources discussed in today's literature.

Similar to allergic reactions in the airways, plasma exudation responses characterise infection-evoked exacerbations of asthma and chronic obstructive pulmonary disease (COPD) [66]. Hence, it seems likely that plasma exudation contributes to the elevated sputum cathelicidin levels reported in COPD patients exhibiting bacterial infection-evoked exacerbations [67].

Human airway in vivo data [64] suggest that plasma exudation mechanisms can be vital for the operation of antimicrobial peptides such as cathelicidins in innate mucosal defence.

\section{Immunoglobulin A}

Balfour-Lynn et al. [68] determined secretory Immunoglobulin A (IgA) as well as total IgA in nasal lavage fluids obtained in infants with upper airway viral infections. During acute infection periods, as compared to symptom-free periods, secretory IgA remained unchanged, whereas total IgA was significantly elevated. The authors considered that plasma exudation was responsible for the increase [68]. This was a rare attention to our then publicised idea that plasma exudation basically represented a non-injurious first-line mucosal defence mechanism [69]. The authoritative contention in those days was otherwise that circulating natural antibodies arrived on the mucosal surface by passive diffusion.
Interestingly, Balfour-Lynn et al. [68] also discussed work where i.v. immunoglobulin had been therapeutically effective in airways infection, presumably arriving at the scene through plasma exudation.

\section{Plasma Exudation and Exudative Hyperresponsiveness at Viral Infection of Human Airways}

\section{Potential Involvement of Many Inducers of Plasma Exudation}

A few examples of airway infection-evoked plasma exudation have been mentioned briefly above. Studies reviewed below involve nasal viral inoculation experiments in human volunteers where plasma exudation has been examined. The epithelium and resident, as well as arriving, leukocytes may produce vasoactive molecules at infective exposures. Mast cells are implicated especially as a source of histamine. There is interest in neutrophil products and especially in the possibility that these cells participate in more sustained plasma leaks $[70,71]$. Once extravasated, additional plasma-derived players, typically kininogen-produced bradykinin [72, 73], may contribute. However, molecules causing plasma exudation in early response to infective agents remain to be determined.

\section{Neurogenic Plasma Leak Does Not Occur in Human} Airways

The popular view that neurogenic plasma leaks are important concerns guinea-pig/rat but not (sic!) human airways. Thus, the neural stimulant capsaicin consistently causes plasma exudation in guinea pig airways [45] but is without this effect in human nasal and bronchial airways, even when given at dose levels causing pain and cough $[52,74,75]$. Hence, it is the non-neural causes of plasma exudation in guinea-pigs that translate into human airways.

\section{Viral Infection-Evoked Plasma Exudation}

Hyperresponsiveness without Increased Absorption

Permeability

Determined as 100-fold increases in fibrinogen concentrations, plasma exudation was associated with cold symptoms after nasal inoculation with human coronavirus 229E. As stated, the data agreed with the possibility that exudation of bulk plasma contributed to resolution of the viral-induced airway disease [76]. Tightness of the mucosal absorption barrier as well as exudative respon- 
siveness to histamine was examined 6 days post-nasal inoculation with the corona virus. At this early phase following resolution of infection and cessation of the associated plasma exudation, the nasal mucosa had maintained its normal barrier tightness determined as absorption of 51Cr-EDTA [54]. However, the concentration-response to histamine (40-400 ug/mL in the nasal pool) was markedly shifted to the left reflecting a significant hyperresponsiveness of the microvascular-epithelial exudation of bulk plasma [54]. These observations demonstrated that the mucosal barrier remained intact, whereas the humoral first-line defence was at an elevated alert following the course of an acute viral infection. Plasma exudative hyperresponsiveness also occurs in active allergic airways disease [77].

\section{Signs Suggesting that Increased Plasma Exudation}

\section{Contributes to Antiviral Resistance}

In atopic individuals, the control plasma exudation response to allergen challenge was greater in a small group of subjects who, at a separate time point, failed to be infected by inoculation with rhinovirus 16 compared to those infected [78]. Other potential defence responses such as inducement of IL-8 were not increased [78]. Furthermore, closely following repeat challenges with large allergen doses, when significant exudation of plasma would have been present, Avila et al. [79] observed that inoculation with rhinovirus was markedly less effective in inducing common cold than when the test subjects had not received allergen challenges. This result was obviously contrary to what the authors expected. However, neither they [79] nor authors of the accompanying editorial [80] took into account that a plasma-exuding mucosa would be expected to exhibit a reduced infectivity. Inferentially, human individuals who are particularly prone to mount an airway mucosal exudation response may also have an improved resistance to common cold infections.

\section{Plasma Exudation in Disease Pathogenesis}

\section{A Tight Mucosal Barrier in Exudative Airways \\ Diseases}

Diseases such as allergic rhinitis and asthma are characterised by plasma exudation [22, 48, 81]. Traditionally, it is taken for granted that plasma exudation is associated with increased absorption permeability in these diseases $[7,44]$. In accord, it is an accepted research paradigm to explain the molecular biology of such a perceived perviousness in vitro and in vivo [44]. However, compelling patient in vivo data are unsupportive regarding the basis for this paradigm.

As reviewed elsewhere [7], numerous carefully controlled in vivo studies have demonstrated unchanged or even reduced rates of absorption of molecules deposited on the airway mucosa in asthma and allergic rhinitis. Different types of absorption tracers have been used to the same effect $[7,82]$. These in vivo data undermine the currently acknowledged teaching that inhaled factors will overtly penetrate the asthmatic and rhinitic mucosa causing and aggravating the disease. Instead it can be concluded, from cumulating patient in vivo data on absorption and exudation, that the definition of asthma and allergic rhinitis as plasma exudation diseases is well compatible with asthma and allergic rhinitis being diseases with maintained mucosal barrier towards inhaled molecules.

\section{Defence Disease Balance: Plasma Exudation in the Epithelial Milieu}

Appearance of plasma proteins in airway surface liquids has traditionally been considered a sign of mucosal oedema. Additionally, plasma "leak" has been regarded as an indication that the epithelial lining must have been injured. These views, which coincide with common descriptions of asthma, may not be valid, at least not without the support of compelling in vivo data obtained under human or human-like conditions. As discussed in this and prior reviews [7], the early plasma exudation response to mucosal challenges is a non-injurious event. It occurs without oedema and the epithelial lining maintains its normal barrier function and structure. As such, plasma exudation qualifies for roles in first-line mucosal defence rather than participating in disease pathogenesis.

On its path to the mucosal surface, a fraction of the extravasated plasma proteins is laid down in intriguing tissue compartments [32]. The exudation process may thus contribute a biologically active milieu in the lamina propria as well as all around epithelial cells in the area of interest. Highly localized, this feature may complement early innate defence activities on the surface. If widespread and sustained, it would contribute to a reactive disease milieu.

Chronic conditions of "plasma leak" may exhibit features distinct from acute defence responses. For example, it could be speculated that it contributes to epithelial fragility. However, even sustained plasma exudation is not necessarily pathogenic. Following asthma-like loss of epithelial cells, occurring without bleeding and leaving an intact basement membrane, there is prompt substitution of the lost barrier with a gel-like formation covering the area of denuded epithelial basement membrane. The gel 
is created by local plasma exudation, which lasts until a new cell cover is re-established over the denuded surface [55]. In the dynamic plasma protein/peptide gel milieu, suited for biological defence and repair, epithelial regeneration proceeds speedily [83]. Hence, thanks to plasma exudation limited sacrifice of epithelial cells may function well as an innate defence mechanism.

Epithelial shedding occurs in human asthma and guinea pig "asthma" but is not known in "mouse asthma" [10]. It is natural to think that epithelial shedding must mean lost barrier function. Yet, as repeatedly demonstrated in patients, asthmatics known to shed epithelial cells exhibit a normal bronchial mucosal barrier towards inhaled molecules [7]. Several factors have been forwarded to explain such a maintained tightness: (A) exceedingly patchy sites of epithelial loss, (B) prompt creation of provisional plasma-derived barriers and $(\mathrm{C})$ high speed of epithelial regeneration from all sides and with all types of bordering epithelial cells dedifferentiating and participating [7]. Hence, asthma as a desquamative disease is compatible with asthma as a disease with a maintained mucosal barrier.

When patches of epithelial denudation abound, epithelial regeneration hot spots become widespread. Then the induced plasma exudation together with other regeneration-induced effects (recruitment and activation of inflammatory cells, mucus secretion and a range of asthmalike airway remodelling effects) has pathogenic potential. These inflammatory and remodelling effects can be induced by epithelial regeneration alone in otherwise healthy airways $[7,84]$. There is also indirect evidence that exaggerated epithelial regeneration may operate as a causative mechanism at inception of asthma as well as in severe asthma $[7,8]$.

\section{Plasma Exudation and "Mucus Plugs"}

Many stimuli, even single autacoids, induce both plasma exudation and mucus secretion [7]. Hence, cooperation between mucus and plasma components in first-line defence of the airway mucosa would be an important area for investigations. Here I will merely make note of pathogenic interactions between mucus and plasma exudate in human bronchi.

In severe asthma, the risk for fungal infection is increased [85]. A worst-case scenario at fungal invasion is complete blockade of segmental airways by plasma (fibrin)- and mucus-rich plugs. In allergic broncho-pulmonary aspergillosis, such plugs can take shape of casts of entire connected branches of bronchi - similar fibrinrich casts occur in plastic bronchitis in children [86, 87]. Plasma exudate may dominate the bronchial plugs/casts

Epithelial Passage of Bulk Plasma that occur following thermoplasty of asthmatic airways likely involving epithelial loss/regeneration [88]. Treatment of the casts involves mechanical removal.

Following lethal RSV infections in infants, the bronchi have been characterized by extensive but patchy denudation of the mucosa - further, fibrin-mucus plugs together with shed epithelial cells have occluded the lumen [89]. This picture agrees with a contributing role of plasma exudation including the involvement of plasma-derived gels [55] shed from numerous epithelial regeneration sites.

In asthma and COPD, the occurrence of 'mucus plugs' contributes to obstruction, especially in severe disease $[90,91]$. Experimental work demonstrating dramatic effects of albumin on mucus swelling [92] and mucus viscosity [93] suggests that even discrete occurrence of plasma exudation may be a contributing factor regarding "mucus plug" formation. Analyses of sputum in asthma [94] and nasal secretions in rhinitis [95] further support involvement of plasma exudation in mucus viscosity. Plasma-derived fibrinogen may serve as a net that keeps lumen contents together. Tethered and viscous secretions, from mucus interacting with exuded plasma, would prevent ciliary clearance and make even extensive coughing and huffing efforts insufficient. Thus, bronchial plugs may form and grow.

\section{Conclusion}

About 150 years ago, Julius Cohnheim noted that extravasated plasma had access to an escape route in mucosal-lined organs, namely, simply seeping through the mucosa (cited in [16]). As discussed here, a swift "seeping" of plasma automatically and promptly occurs at mucosal exudative challenges. The passage of bulk plasma between plasticity-yielding epithelial junctions counteracts oedema production and does not reduce the normal barrier function of the epithelial lining. The early appearance of the armamentarium of plasma protein systems on the challenged surface of an intact airway mucosa would serve a main purpose of warding off threatening insults before penetration has occurred into the tissue. This humoral innate defence opportunity of mucosal front lines operates in vivo in humans.

\section{Acknowledgement}

I thank Dr. Cecilia Andersson for Figure 1. 


\section{Disclosure Statement}

The authors declare that they have no conflicts of interest to disclose.

\section{Funding Sources}

There is no funding to declare.

\section{References}

1 Iwasaki A, Foxman EF, Molony RD. Early local immune defences in the respiratory tract. Nat Rev Immunol. 2017 Jan;17(1):7-20.

2 Hartl D, Tirouvanziam R, Laval J, Greene CM, Habiel D, Sharma L, et al. Innate Immunity of the Lung: From Basic Mechanisms to Translational Medicine. J Innate Immun. 2018;10(5-6):487-501.

3 Persson CG. Centennial notions of asthma as an eosinophilic, desquamative, exudative, and steroid-sensitive disease. Lancet. 1997 Oct:350(9083):1021-4.

4 Persson C, Uller L. HH Salter (1860s): taking cold as original cause and provocative of attacks of asthma. Thorax. 2013 May;68(5):489.

5 Persson C. Clinical research, or classical clinical research? Nat Med. 1999 Jul;5(7):714-5.

6 Persson C. Discoveries in complex biosystems. Nat Biotechnol. 1997 Oct;15(10):927.

7 Persson C. Airways exudation of plasma macromolecules: innate defense, epithelial regeneration, and asthma. J Allergy Clin Immunol. 2019 Apr;143(4):1271-86.

8 Persson C. In vivo observations provide insight into roles of eosinophils and epithelial cells in asthma. Eur Respir J. 2019 Oct;54(4): 1900470.

9 Persson CG, Erjefält JS, Korsgren M, Sundler F. The mouse trap. Trends Pharmacol Sci. 1997 Dec;18(12):465-7.

10 Persson CG. Con: mice are not a good model of human airway disease. Am J Respir Crit Care Med. 2002 Jul;166(1):6-7; discussion 8.

11 Ekdahl KN, Teramura Y, Hamad OA, Asif S, Duehrkop C, Fromell K, et al. Dangerous liaisons: complement, coagulation, and kallikrein/kinin cross-talk act as a linchpin in the events leading to thromboinflammation. Immunol Rev. 2016 Nov;274(1):245-69.

12 Dzik S. Complement and Coagulation: Cross Talk Through Time. Transfus Med Rev. 2019 Oct;33(4):199-206.

13 Allen RJ, Byrnes AP. Interaction of adenovirus with antibodies, complement, and coagulation factors. FEBS Lett. 2019 Dec;593(24): 3449-60.

14 Svensjö E, Arfors KE, Raymond RM, Grega GJ. Morphological and physiological correlation of bradykinin-induced macromolecular efflux. Am J Physiol. 1979 Apr;236(4): H600-6.

15 Persson CG, Erjefält I, Grega GJ, Svensjö E. The role of beta-receptor agonists in the inhibition of pulmonary edema. Ann N Y Acad Sci. 1982;384:544-57.

16 Persson CG. Plasma exudation and asthma. Lung. 1988;166(1):1-23.
17 Wagner EM, Jenkins J, Schmieder A, Eldridge L, Zhang Q, Moldobaeva A, et al. Angiogenesis and airway reactivity in asthmatic Brown Norway rats. Angiogenesis. 2015 Jan; 18(1): $1-11$.

18 Irvin CG, Bates JH. Physiologic dysfunction of the asthmatic lung: what's going on down there, anyway? Proc Am Thorac Soc. 2009 May;6(3):306-11.

19 Yuda H, Adachi Y, Taguchi O, Gabazza EC, Hataji O, Fujimoto H, et al. Activated protein C inhibits bronchial hyperresponsiveness and Th2 2 cytokine expression in mice. Blood. 2004 Mar;103(6):2196-204.

20 Erjefält JS, Andersson P, Gustafsson B, Korsgren M, Sonmark B, Persson CG. Allergen challenge-induced extravasation of plasma in mouse airways. Clin Exp Allergy. 1998 Aug; 28(8):1013-20.

21 Svensjö E, Persson CG, Rutili G. Inhibition of bradykinin induced macromolecular leakage from post-capillary venules by a beta2-adrenoreceptor stimulant, terbutaline. Acta Physiol Scand. 1977 Dec;101(4):504-6.

22 Persson CG. Role of plasma exudation in asthmatic airways. Lancet. 1986 Nov;2(8516): 1126-9.

23 Persson CG. The action of beta-receptors on microvascular endothelium or: is airways plasma exudation inhibited by beta-agonists? Life Sci. 1993;52(26):2111-21.

24 Greiff L, Wollmer P, Andersson M, Svensson C, Persson CG. Effects of formoterol on histamine induced plasma exudation in induced sputum from normal subjects. Thorax. 1998 Dec;53(12):1010-3.

25 Svensson C, Alkner U, Pipkorn U, Persson CG. Histamine-induced airway mucosal exudation of bulk plasma and plasma-derived mediators is not inhibited by intravenous bronchodilators. Eur J Clin Pharmacol. 1994; 46(1):59-65.

26 Greiff L, Andersson M, Svensson C, Alkner U, Persson CG. Glucocorticoids may not inhibit plasma exudation by direct vascular antipermeability effects in human airways. Eur Respir J. 1994 Jun;7(6):1120-4.

27 Grega GJ, Adamski SW. The role of venular endothelial cells in the regulation of macromolecular permeability. Microcirc Endothelium Lymphatics. 1988 Apr;4(2):143-67.

28 Persson CG, Erjefält I, Andersson P. Leakage of macromolecules from guinea-pig tracheobronchial microcirculation. Effects of allergen, leukotrienes, tachykinins, and anti-asthma drugs. Acta Physiol Scand. 1986 May; 127(1):95-105.
29 Rogers DF, Alton EW, Aursudkij B, Boschetto P, Dewar A, Barnes PJ. Effect of platelet activating factor on formation and composition of airway fluid in the guinea-pig trachea. J Physiol. 1990 Dec;431(1):643-58.

30 Erjefalt I, Luts A, Persson CG. Appearance of airway absorption and exudation tracers in guinea pig tracheobronchial lymph nodes. J Appl Physiol (1985). 1993 Feb;74(2):817-24.

31 Luts A, Sundler F, Erjefält I, Persson CG. The airway epithelial lining in guinea pigs is intact promptly after the mucosal crossing of a large amount of plasma exudate. Int Arch Allergy Appl Immunol. 1990;91(4):385-8.

32 Erjefält JS, Erjefält I, Sundler F, Persson CG. Epithelial pathways for luminal entry of bulk plasma. Clin Exp Allergy. 1995 Feb;25(2): 187-95.

33 Serikov VB, Jang YJ, Widdicombe JH. Estimate of the subepithelial hydrostatic pressure that drives inflammatory transudate into the airway lumen. J Appl Physiol (1985). 2002 Apr;92(4):1702-8.

34 Erjefält IA, Wagner ZG, Strand SE, Persson CG. A method for studies of tracheobronchial microvascular permeability to macromolecules. J Pharmacol Methods. 1985 Dec;14(4): 275-83.

35 Erjefält I, Persson CG. Inflammatory passage of plasma macromolecules into airway wall and lumen. Pulm Pharmacol. 1989;2(2):93102.

36 Erjefält I, Persson CG. Increased sensitivity to toluene diisocyanate (TDI) in airways previously exposed to low doses of TDI. Clin Exp Allergy. 1992 Sep;22(9):854-62.

37 Erjefält I, Greiff L, Alkner U, Persson CG. Allergen-induced biphasic plasma exudation responses in guinea pig large airways. Am Rev Respir Dis. 1993 Sep;148(3):695-701.

38 Greiff L, Erjefält I, Erjefält JS, Wollmer P, Persson CG. Effects of hydrogen peroxide on the guinea-pig tracheobronchial mucosa in vivo. Acta Physiol Scand. 1999 Apr;165(4): 415-20.

39 Persson CG, Erjefält I. Inflammatory leakage of macromolecules from the vascular compartment into the tracheal lumen. Acta Physiol Scand. 1986 Apr;126(4):615-6.

40 Azizi F, Matsumoto PS, Wu DX, Widdicombe $\mathrm{JH}$. Effects of hydrostatic pressure on permeability of airway epithelium. Exp Lung Res. 1997 May-Jun;23(3):257-67.

41 Tokuda S, Miyazaki H, Nakajima K, Yamada T, Marunaka Y. Hydrostatic pressure regulates tight junctions, actin cytoskeleton and transcellular ion transport. Biochem Biophys Res Commun. 2009 Dec;390(4):1315-21. 
42 Persson CG, Erjefält I, Gustafsson B, Luts A. Subepithelial hydrostatic pressure may regulate plasma exudation across the mucosa. Int Arch Allergy Appl Immunol. 1990;92(2): 148-53.

43 Gustafsson BG, Persson CG. Asymmetrical effects of increases in hydrostatic pressure on macromolecular movement across the airway mucosa. A study in guinea-pig tracheal tube preparations. Clin Exp Allergy. 1991 Jan; 21(1):121-6.

44 Georas SN, Rezaee F. Epithelial barrier function: at the front line of asthma immunology and allergic airway inflammation. J Allergy Clin Immunol. 2014 Sep;134(3):509-20.

45 Erjefält I, Persson CG. Allergen, bradykinin, and capsaicin increase outward but not inward macromolecular permeability of guinea-pig tracheobronchial mucosa. Clin Exp Allergy. 1991 Mar;21(2):217-24.

46 Persson CG, Gustafsson B, Erjefält JS, Sundler F. Mucosal exudation of plasma is a noninjurious intestinal defense mechanism. Allergy. 1993 Nov;48(8):581-6.

47 Gustafsson B, Persson CG. Allergen-induced mucosal exudation of plasma into rat ileum and its inhibition by budesonide. Scand J Gastroenterol. 1992 Jul;27(7):587-93.

48 Persson CG, Svensson C, Greiff L, Anderson $\mathrm{M}$, Wollmer P, Alkner U, et al. The use of the nose to study the inflammatory response of the respiratory tract. Thorax. 1992 Dec; 47(12):993-1000.

49 Greiff L, Pipkorn U, Alkner U, Persson CG. The 'nasal pool' device applies controlled concentrations of solutes on human nasal airway mucosa and samples its surface exudations/secretions. Clin Exp Allergy. 1990 May; 20(3):253-9.

50 Salomonsson P, Grönneberg R, Gilljam H, Andersson O, Billing B, Enander I, et al. Bronchial exudation of bulk plasma at allergen challenge in allergic asthma. Am Rev Respir Dis. 1992 Dec;146(6): 1535-42.

51 Svensson C, Grönneberg R, Andersson M, Alkner U, Andersson O, Billing B, et al. Allergen challenge-induced entry of alpha 2-macroglobulin and tryptase into human nasal and bronchial airways. J Allergy Clin Immunol. 1995 Aug;96(2):239-46.

52 Greiff L, Andersson M, Coman WB, Lindberg $\mathrm{H}$, Marko-Varga G, Wallwork B, et al. Challenge-induced plasma exudation and mucinous secretion in human airways. Clin Physiol Funct Imaging. 2005 Jul;25(4):241-5.

53 Svensson C, Baumgarten CR, Pipkorn U, Alkner U, Persson CG. Reversibility and reproducibility of histamine induced plasma leakage in nasal airways. Thorax. 1989 Jan;44(1): 13-8.

54 Greiff L, Andersson M, Akerlund A, Wollmer $\mathrm{P}$, Svensson C, Alkner U, et al. Microvascular exudative hyperresponsiveness in human coronavirus-induced common cold. Thorax. 1994 Feb;49(2):121-7.
55 Erjefält JS, Erjefält I, Sundler F, Persson CG. Microcirculation-derived factors in airway epithelial repair in vivo. Microvasc Res. 1994 Sep;48(2):161-78.

56 Greiff L, Andersson M, Erjefält JS, Svensson C, Persson CG. Loss of size-selectivity at histamine-induced exudation of plasma proteins in atopic nasal airways. Clin Physiol Funct Imaging. 2002 Jan;22(1):28-31.

57 Greiff L, Wollmer P, Pipkorn U, Persson CG. Absorption of 51Cr EDTA across the human nasal airway barriers in the presence of topical histamine. Thorax. 1991 Sep;46(9):630-2.

58 Persson C, Uller L. Resolution of leucocytemediated mucosal diseases. A novel in vivo paradigm for drug development. Br J Pharmacol. 2012 Apr;165(7):2100-9.

59 Liu L, Mul FP, Kuijpers TW, Lutter R, Roos D, Knol EF. Neutrophil transmigration across monolayers of endothelial cells and airway epithelial cells is regulated by different mechanisms. Ann N Y Acad Sci. 1996 Oct 31;796: 21-9.

60 Krug N, Tschernig T, Erpenbeck VJ, Hohlfeld JM, Köhl J. Complement factors C3a and C5a are increased in bronchoalveolar lavage fluid after segmental allergen provocation in subjects with asthma. Am J Respir Crit Care Med. 2001 Nov;164(10 Pt 1):1841-3.

61 Thwaites RS, Gunawardana NC, Broich V, Mann EH, Ahnström J, Campbell GA, et al. Biphasic activation of complement and fibrinolysis during the human nasal allergic response. J Allergy Clin Immunol. 2018 May; 141(5):1892-1895.e6.

62 Andersson M, Michel L, Llull JB, Pipkorn U. Complement activation on the nasal mucosal surface-a feature of the immediate allergic reaction in the nose. Allergy. 1994 Apr;49(4): $242-5$.

63 Kicic A, Hallstrand TS, Sutanto EN, Stevens PT, Kobor MS, Taplin C, et al. Decreased fibronectin production significantly contributes to dysregulated repair of asthmatic epithelium. Am J Respir Crit Care Med. 2010 May;181(9):889-98.

64 Liu MC, Xiao HQ, Brown AJ, Ritter CS Schroeder J. Association of vitamin D and antimicrobial peptide production during latephase allergic responses in the lung. Clin Exp Allergy. 2012 Mar;42(3):383-91.

65 Sørensen O, Cowland JB, Askaa J, Borregaard N. An ELISA for hCAP-18, the cathelicidin present in human neutrophils and plasma. J Immunol Methods. 1997 Aug;206(1-2):53-9.

66 Persson C, Uller L. Roles of plasma exudation in asthma and COPD. Clin Exp Allergy. 2009 Nov;39(11):1626-9.

67 Parameswaran GI, Sethi S, Murphy TF. Effects of bacterial infection on airway antimicrobial peptides and proteins in COPD. Chest. 2011 Sep;140(3):611-7.

68 Balfour-Lynn IM, Valman B, Silverman M, Webster AD. Nasal IgA response in wheezy infants. Arch Dis Child. 1993 Apr;68(4): $472-6$.
69 Persson CG, Erjefält I, Alkner U, Baumgarten C, Greiff L, Gustafsson B, et al. Plasma exudation as a first line respiratory mucosal defence. Clin Exp Allergy. 1991 Jan;21(1):1724.

70 Edens HA, Parkos CA. Neutrophil transendothelial migration and alteration in vascular permeability: focus on neutrophil-derived azurocidin. Curr Opin Hematol. 2003 Jan; 10(1):25-30

71 Ma Y, Yang X, Chatterjee V, Meegan JE, Beard RS Jr, Yuan SY. Role of Neutrophil Extracellular Traps and Vesicles in Regulating Vascular Endothelial Permeability. Front Immunol. 2019 May; 10:1037.

72 Proud D. The kinin system in rhinitis and asthma. Clin Rev Allergy Immunol. 1998; 16(4):351-64.

73 Imamura T, Tanase S, Szmyd G, Kozik A, Travis J, Potempa J. Induction of vascular leakage through release of bradykinin and a novel kinin by cysteine proteinases from Staphylococcus aureus. J Exp Med. 2005 May;201(10): 1669-76.

74 Rajakulasingam K, Polosa R, Lau LC, Church MK, Holgate ST, Howarth PH. Nasal effects of bradykinin and capsaicin: influence on plasma protein leakage and role of sensory neurons. J Appl Physiol (1985). 1992 Apr; 72(4):1418-24

75 Greiff L, Svensson C, Andersson M, Persson CG. Effects of topical capsaicin in seasonal allergic rhinitis. Thorax. 1995 Mar;50(3):225-9.

76 Akerlund A, Greiff L, Andersson M, Bende M, Alkner U, Persson CG. Mucosal exudation of fibrinogen in coronavirus-induced common colds. Acta Otolaryngol. 1993 Sep;113(5): 642-8.

77 Meyer P, Andersson M, Persson CG, Greiff L. Steroid-sensitive indices of airway inflammation in children with seasonal allergic rhinitis Pediatr Allergy Immunol. 2003 Feb;14(1):605 .

78 Greiff L, Andersson M, Svensson C, Linden M, Myint S, Persson CG. Allergen challengeinduced acute exudation of IL-8, ECP and alpha2-macroglobulin in human rhinovirusinduced common colds. Eur Respir J. 1999 Jan;13(1):41-7.

79 Avila PC, Abisheganaden JA, Wong H, Liu J, Yagi S, Schnurr D, et al. Effects of allergic inflammation of the nasal mucosa on the severity of rhinovirus 16 cold. J Allergy Clin Immunol. 2000 May;105(5):923-32.

80 Busse WW, Gern JE. Do allergies protect against the effects of a rhinovirus cold? J Allergy Clin Immunol. 2000 May;105(5):88991.

81 Dunnill MS. The pathology of asthma, with special reference to changes in the bronchial mucosa. J Clin Pathol. 1960 Jan;13(1):27-33.

82 Georas S, Ransom N, Hillman S, Eliseeva S, Veazey J, Smyth T, et al. The leaky lung test: A pilot study using inhaled mannitol to measure airway barrier function in asthma. J Asthma. 2019 Dec;56(12):1257-65. 
83 Erjefält JS, Erjefält I, Sundler F, Persson CG. In vivo restitution of airway epithelium. Cell Tissue Res. 1995 Aug;281(2):305-16.

84 Persson CG, Erjefält JS, Erjefält I, Korsgren MC, Nilsson MC, Sundler F. Epithelial shedding-restitution as a causative process in airway inflammation. Clin Exp Allergy. 1996 Jul; 26(7):746-55.

85 Li E, Landers CT, Tung HY, Knight JM, Marshall Z, Luong AU, et al. Fungi in Mucoobstructive Airway Diseases. Ann Am Thorac Soc. 2018 Nov;15(Suppl 3):S198-204.

86 Brogan TV, Finn LS, Pyskaty DJ Jr, Redding GJ, Ricker D, Inglis A, et al. Plastic bronchitis in children: a case series and review of the medical literature. Pediatr Pulmonol. 2002 Dec;34(6):482-7.

87 Panchabhai TS, Mukhopadhyay S, Sehgal S, Bandyopadhyay D, Erzurum SC, Mehta AC. Plugs of the Air Passages: A Clinicopathologic Review. Chest. 2016 Nov;150(5):1141-57.
88 Facciolongo N, Menzella F, Lusuardi M, Piro $\mathrm{R}$, Galeone C, Castagnetti C, et al. Recurrent lung atelectasis from fibrin plugs as a very early complication of bronchial thermoplasty: a case report. Multidiscip Respir Med. 2015 Mar;10(1):9.

89 Johnson JE, Gonzales RA, Olson SJ, Wright PF, Graham BS. The histopathology of fatal untreated human respiratory syncytial virus infection. Mod Pathol. 2007 Jan;20(1):10819.

90 Ramos FL, Krahnke JS, Kim V. Clinical issues of mucus accumulation in COPD. Int J Chron Obstruct Pulmon Dis. 2014 Jan;9:139-50.

91 Dunican EM, Elicker BM, Gierada DS, Nagle SK, Schiebler ML, Newell JD, et al.; National Heart Lung and Blood Institute (NHLBI) Severe Asthma Research Program (SARP). Mucus plugs in patients with asthma linked to eosinophilia and airflow obstruction. J Clin Invest. 2018 Mar;128(3):997-1009.
92 Aitken ML, Verdugo P. Donnan mechanism of mucin release and conditioning in goblet cells: the role of polyions. Symp Soc Exp Biol. 1989;43:73-80.

93 List SJ, Findlay BP, Forstner GG, Forstner JF. Enhancement of the viscosity of mucin by serum albumin. Biochem J. 1978 Nov;175(2): $565-71$.

94 Innes $\mathrm{AL}$, Carrington SD, Thornton DJ, Kirkham S, Rousseau K, Dougherty RH, et al. Ex vivo sputum analysis reveals impairment of protease-dependent mucus degradation by plasma proteins in acute asthma. Am J Respir Crit Care Med. 2009 Aug;180(3):203-10.

95 Takabayashi T, Imoto Y, Sakashita M, Kato Y, Tokunaga T, Yoshida K, et al. Nattokinase, profibrinolytic enzyme, effectively shrinks the nasal polyp tissue and decreases viscosity of mucus. Allergol Int. 2017 Oct;66(4):594602. 